\title{
Prediction-Based Compensation for Gate On/Off Latency during Respiratory-Gated Radiotherapy
}

\author{
Hisashi Johno $\mathbb{D D}^{1,2}$ Masahide Saito ${ }^{1 D}{ }^{2}$ and Hiroshi Onishi ${ }^{2}$ \\ ${ }^{1}$ Department of Mathematical Sciences, University of Yamanashi, Japan \\ ${ }^{2}$ Department of Radiology, University of Yamanashi, Japan \\ Correspondence should be addressed to Hisashi Johno; johnoh@yamanashi.ac.jp
}

Received 3 August 2018; Accepted 8 October 2018; Published 27 November 2018

Academic Editor: Chuangyin Dang

Copyright ( $\odot 2018$ Hisashi Johno et al. This is an open access article distributed under the Creative Commons Attribution License, which permits unrestricted use, distribution, and reproduction in any medium, provided the original work is properly cited.

During respiratory-gated radiotherapy (RGRT), gate on and off latencies cause deviations of gating windows, possibly leading to delivery of low- and high-dose radiations to tumors and normal tissues, respectively. Currently, there are no RGRT systems that have definite tools to compensate for the delays. To address the problem, we propose a framework consisting of two steps: (1) multistep-ahead prediction and (2) prediction-based gating. For each step, we have devised a specific algorithm to accomplish the task. Numerical experiments were performed using respiratory signals of a phantom and ten volunteers, and our prediction-based RGRT system exhibited superior performance in more than a few signal samples. In some, however, signal prediction and prediction-based gating did not work well, maybe due to signal irregularity and/or baseline drift. The proposed approach has potential applicability in RGRT, and further studies are needed to verify and refine the constituent algorithms.

\section{Introduction}

Respiratory-gated radiotherapy (RGRT) is a widely employed means of treating tumors that move with respiration [1-3]. In RGRT, radiation is administered within particular phases of the patient's breathing cycle (called as gating windows), which are determined by monitoring respiratory motion in the form of a respiratory signal using either external or internal markers. Note that, although there are some options for RGRT (e.g., whether to choose amplitude-based or phase-based gating and whether to gate during inhalation or exhalation), this study focuses only on amplitude-based gating during exhalation, which is a common setting in clinical practice. Several RGRT systems have been developed, and some take considerable time from the detection of a signal change to the execution of a gate on/off command (Table 1). The gate on/off latency causes deviations of gating windows in conventional RGRT (Figure 1), possibly leading to delivery of low- and high-dose radiation to tumor and normal tissues, respectively. At present, there are no RGRT systems that have definite techniques to compensate for the delays. Therefore, here, we propose a prediction-based system to address the problem.
This paper is organized as follows. The devised framework is described in Section 2, experimental results are in Section 3, and the conclusions follow in Section 4.

\section{Methods}

In this section, we describe our new approach to compensate for gate on/off latency. This consists of two steps: (1) multistep-ahead prediction and (2) prediction-based gating.

2.1. Multistep-Ahead Prediction. Several prediction algorithms for respiratory signals have been proposed, and most of them adopt single-output strategies [7, 8]. However, in our framework, multiple-output multistep-ahead prediction is required. Therefore, we have devised an algorithm for this purpose.

A respiratory signal is regarded as a sequence

$$
\left\{x_{t}\right\}, t=0,1,2, \ldots,
$$

of equally spaced time-series observations in a space $\chi$, with a time interval of $\Delta \tau$ seconds (s), where $\Delta \tau>0$. Let $n$ and $m$ be positive integers. For each time point $t \geq n$, multistep-ahead 
TABLE 1: Gate on and off latencies of some gating systems.

\begin{tabular}{lcccc}
\hline Monitor & Linac & Gate on delay & Gate off delay & Reference \\
\hline Abches (APEX) & Elekta Synergy & $336 \mathrm{~ms}$ & $88 \mathrm{~ms}$ & {$[4]$} \\
AlignRT (VisionRT) & Varian Clinac iX & $356 \mathrm{~ms}$ & $529 \mathrm{~ms}$ & $60 \mathrm{~ms}$ \\
Calypso (Varian) & Varian Clinac iX & $209 \mathrm{~ms}$ & {$[5]$} \\
Catalyst (C-RAD) & Elekta Synergy & $851 \mathrm{~ms}$ & $215 \mathrm{~ms}$ & {$[5]$} \\
\hline
\end{tabular}

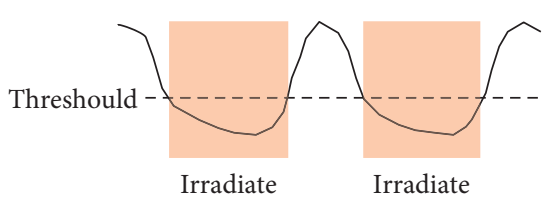

(a)

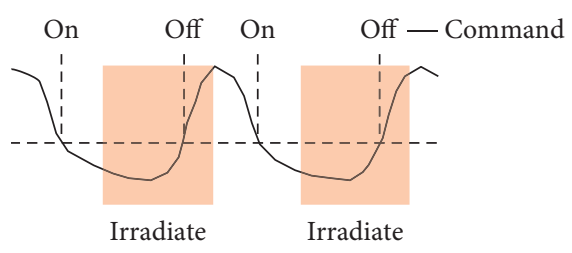

(b)

FIGURE 1: Problem with conventional RGRT. (a) Given a respiratory signal and a gating threshold, gating windows should ideally be the time when the signal is lower than the threshold. (b) In conventional RGRT, gate on and off commands are sent just when the signal is lower and higher than the threshold, respectively. Hence, gate on and off delays cause deviations of gating windows.

prediction aims to forecast the $m$-tuple $\left(x_{t}, \ldots, x_{t+m-1}\right)$ of subsequent observations, given the previous $n$-tuple $\left(x_{t-n}, \ldots, x_{t-1}\right)$. Hence, our goal here is to form a predictor mapping on $\chi^{n}$ to $\chi^{m}$. Suppose $\chi^{n}$ is a metric space with a metric $d_{n}$. Let us have a learning set $\mathscr{L}=\left\{\left(\widetilde{\mathbf{x}}_{i}, \widetilde{\mathbf{y}}_{i}\right) \in \chi^{n} \times \chi^{m}\right\}$, where $i$ ranges over some finite totally ordered set (see Section 2.3 for an example of the learning set preparation). Then, for a test tuple $\mathbf{x} \in \chi^{n}$, we predict the next $m$-tuple as

$$
\Psi_{\mathscr{L}}(\mathbf{x})=\widetilde{\mathbf{y}}_{p}
$$

where $p$ is the largest index such that $d_{n}\left(\mathbf{x}, \widetilde{\mathbf{x}}_{p}\right) \leq d_{n}\left(\mathbf{x}, \widetilde{\mathbf{x}}_{i}\right)$ for all $i$. Throughout this paper, we suppose that $\chi=\mathbb{R}$, and $\chi^{k}$, which equals $\mathbb{R}^{k}(k=1,2,3, \ldots)$, is a real $k$-space with the Euclidean metric, i.e.,

$$
d_{k}(\mathbf{a}, \mathbf{b})=\sqrt{\sum_{j=0}^{k-1}\left(a_{j}-b_{j}\right)^{2}} \quad\left(\begin{array}{l}
\mathbf{a}=\left(a_{0}, \ldots, a_{k-1}\right) \in \mathbb{R}^{k} \\
\mathbf{b}=\left(b_{0}, \ldots, b_{k-1}\right) \in \mathbb{R}^{k}
\end{array}\right) .
$$

2.2. Prediction-Based RGRT. Let $x_{t} \in \mathbb{R}^{1}(t \geq n)$ be the current observation, $\beta \in \mathbb{R}^{1}$ be a gating threshold, and $m_{1}$ and $m_{0}$ be the numbers of time points corresponding to gate on and off delays, respectively. Given learning sets $\mathscr{L}_{1} \subset \mathbb{R}^{n} \times \mathbb{R}^{2 m_{1}+1}$ and $\mathscr{L}_{0} \subset \mathbb{R}^{n} \times \mathbb{R}^{2 m_{0}+1}$ (see Section 2.3 for an example of the learning set construction), the function $G_{\mathscr{L}_{1}, \mathscr{L}_{0}}$ defined below is used for a prediction-based gating.

(1) Case $m_{1} \geq m_{0}$ :

$$
G_{\mathscr{L}_{1}, \mathscr{L}_{0}}(t, \beta)=\left\{\begin{array}{cc}
1, & \text { if } \xi_{2 m_{1}+1, \beta}\left(\Psi_{\mathscr{L}_{1}}\left(\mathbf{x}_{t}\right)\right)<0 \text { or } \\
& \xi_{2 m_{0}+1, \beta}\left(\Psi_{\mathscr{L}_{0}}\left(\mathbf{x}_{t}\right)\right)<0, \\
0, & \text { otherwise. }
\end{array}\right.
$$

(2) Case $m_{1}<m_{0}$ :

$$
G_{\mathscr{L}_{1}, \mathscr{L}_{0}}(t, \beta)=\left\{\begin{array}{cc}
1, & \text { if } \xi_{2 m_{1}+1, \beta}\left(\Psi_{\mathscr{L}_{1}}\left(\mathbf{x}_{t}\right)\right)<0 \text { and } \\
& \xi_{2 m_{0}+1, \beta}\left(\Psi_{\mathscr{L}_{0}}\left(\mathbf{x}_{t}\right)\right)<0, \\
0, & \text { otherwise, }
\end{array}\right.
$$

where $\xi_{m, \beta}: \mathbb{R}^{m} \longrightarrow \mathbb{Z}$ (the set of integers) is defined by

$$
\xi_{m, \beta}\left(\left(a_{0}, \ldots, a_{m-1}\right)\right)=\sum_{k=0}^{m-1} \operatorname{sgn}\left(a_{k}-\beta\right),
$$

and $\mathbf{x}_{t}=\left(x_{t-n}, \ldots, x_{t-1}\right) \in \mathbb{R}^{n}$. Note that sgn $: \mathbb{R}^{1} \longrightarrow$ $\{-1,0,1\}$ denotes the signum function, i.e.,

$$
\operatorname{sgn}(a)=\left\{\begin{array}{cl}
1, & \text { if } a>0, \\
0, & \text { if } a=0, \\
-1, & \text { if } a<0 .
\end{array}\right.
$$

In our prediction-based RGRT system (pRGRT), gate on command is sent if $G_{\mathscr{L}_{1}, \mathscr{L}_{0}}(t, \beta)=1$, while gate off command is sent if $G_{\mathscr{L}_{1}, \mathscr{L}_{0}}(t, \beta)=0$.

2.3. Construction of a Learning Set. To begin with, a respiratory signal tuple $\left(x_{0}, \ldots, x_{N-1}\right) \in \mathbb{R}^{N}$ is smoothed using the finite Fourier transform [9]. In detail, the mapping $\Phi_{\alpha, N}: \mathbb{R}^{N} \longrightarrow \mathbb{R}^{N}\left(\alpha \in \mathbb{R}^{1}\right)$ defined below is applied for the smoothing.

$$
\begin{aligned}
\Phi_{\alpha, N}\left(\left(x_{0}, \ldots, x_{N-1}\right)\right) & =\left(\widetilde{x}_{0}, \ldots, \tilde{x}_{N-1}\right), \\
\left(s_{0}, \ldots, s_{N-1}\right) & =\mathscr{F}_{N}\left(W\left(\left(x_{0}, \ldots, x_{N-1}\right)\right)\right), \\
\left(u_{0}, \ldots, u_{N-1}\right) & =\mathscr{F}_{N}^{-1}\left(F_{\alpha}\left(\left(s_{0}, \ldots, s_{N-1}\right)\right)\right), \\
\left(\tilde{x}_{0}, \ldots, \widetilde{x}_{N-1}\right) & =W^{-1}\left(R\left(\left(u_{0}, \ldots, u_{N-1}\right)\right)\right),
\end{aligned}
$$


where $\mathscr{F}_{N}$ is the finite Fourier transform on $\mathbb{C}^{N}$ (a complex $N$-space) defined by

$$
\begin{aligned}
& \mathscr{F}_{N}\left(\left(a_{0}, \ldots, a_{N-1}\right)\right)=\left(\widehat{a}_{0}, \ldots, \widehat{a}_{N-1}\right), \\
& \widehat{a}_{k}=\sum_{j=0}^{N-1} a_{j} \exp \left(-\frac{2 \pi \sqrt{-1} j k}{N}\right) \\
& \quad(k=0, \ldots, N-1),
\end{aligned}
$$

while its inverse is given by

$$
\begin{aligned}
& \mathscr{F}_{N}^{-1}\left(\left(a_{0}, \ldots, a_{N-1}\right)\right)=\left(\check{a}_{0}, \ldots, \check{a}_{N-1}\right), \\
& \check{a}_{k}=\frac{1}{N} \sum_{j=0}^{N-1} a_{j} \exp \left(\frac{2 \pi \sqrt{-1} j k}{N}\right) \\
&(k=0, \ldots, N-1),
\end{aligned}
$$

$$
\begin{array}{r}
W: \mathbb{R}^{N} \longrightarrow \mathbb{R}^{N} \text { is defined by } \\
W\left(\left(x_{0}, \ldots, x_{N-1}\right)\right)=\left(w_{0} x_{0}, \ldots, w_{N-1} x_{N-1}\right), \\
w_{k}=0.54-0.46 \cos \left(\frac{2 \pi k}{N-1}\right) \\
\quad(k=0, \ldots, N-1),
\end{array}
$$

while its inverse is given by

$$
W^{-1}\left(\left(x_{0}, \ldots, x_{N-1}\right)\right)=\left(\frac{x_{0}}{w_{0}}, \ldots, \frac{x_{N-1}}{w_{N-1}}\right),
$$

$F_{\alpha}: \mathbb{C}^{N} \longrightarrow \mathbb{C}^{N}$ is defined by

$$
F_{\alpha}\left(\left(s_{0}, \ldots, s_{N-1}\right)\right)=\left(\widetilde{s}_{0}, \ldots, \widetilde{s}_{N-1}\right),
$$

$\widetilde{s}_{k}=\left\{\begin{array}{ll}0, & \text { if }\left|k-\frac{N}{2}\right|<\frac{N}{2}-\alpha, \\ s_{k}, & \text { otherwise, }\end{array} \quad(k=0, \ldots, N-1)\right.$,

and $R: \mathbb{C}^{N} \longrightarrow \mathbb{R}^{N}$ is by

$$
R\left(\left(u_{0}, \ldots, u_{N-1}\right)\right)=\left(\operatorname{Re}\left(u_{0}\right), \ldots, \operatorname{Re}\left(u_{N-1}\right)\right) .
$$

Note that $W$ defined above is called the Hamming window [10]. The parameter $\alpha \in \mathbb{R}^{1}$ can be set freely, e.g., we set

$$
\alpha=N \Delta \tau f \quad\left(0 \leq f \leq \frac{1}{2 \Delta \tau}\right),
$$

to filter out signal components with frequencies larger than $f$ hertz $(\mathrm{Hz})$.

For a signal tuple $\left(x_{0}, \ldots, x_{N-1}\right) \in \mathbb{R}^{N}$,

$$
\left(\tilde{x}_{0}, \ldots, \tilde{x}_{N-1}\right)=\Phi_{\alpha, N}\left(\left(x_{0}, \ldots, x_{N-1}\right)\right),
$$

is called the smoothed signal tuple and used to construct a learning set $\left\{\left(\tilde{x}_{i}, \tilde{y}_{i}\right)\right\} \subset \mathbb{R}^{n} \times \mathbb{R}^{m}(n+m \leq N)$ by putting

$$
\begin{aligned}
\widetilde{\mathbf{x}}_{i} & =\left(\widetilde{x}_{i}, \ldots, \widetilde{x}_{i+n-1}\right), \\
\widetilde{\mathbf{y}}_{i} & =\left(\widetilde{x}_{i+n}, \ldots, \widetilde{x}_{i+n+m-1}\right),
\end{aligned}
$$

for $i=0, \ldots, N-n-m$.

\section{Numerical Results and Discussion}

To validate the devised algorithms, respiratory signals of a dynamic thoracic phantom (CIRS, Virginia, USA) and ten healthy volunteers were measured with Abches (APEX Medical, Inc., Tokyo, Japan), which is a respirationmonitoring device developed by Onishi et al. [11] and routinely used in our university hospital. Note that, for simplicity, we supposed that $\Delta \tau=0.03$ although the actual time intervals were not precisely equal to $0.03 \mathrm{~s}$. Signal values were given in the unit of $\mathrm{mm}$.

3.1. Smoothing of a Respiratory Signal. To test the algorithm of smoothing a respiratory signal, the phantom's signal was measured for $20 \mathrm{~s}$ (667 time points) and an artificial noise was added (13.65-13.7 s), forming a signal tuple $\mathbf{x}=\left(x_{0}, \ldots, x_{N-1}\right)$. Then $\Phi_{\alpha, N}(\mathbf{x})$ was calculated (Equations (8a)-(8d)), setting $\alpha=N \Delta \tau$ to filter out high frequency $(>1 \mathrm{~Hz})$ components. As shown in Figure 2, we succeeded in removing noisy components of $\mathbf{x}$.

3.2. Prediction of a Respiratory Signal. The prediction algorithm was tested using respiratory signals of ten volunteers, measured for $300 \mathrm{~s}$ (10000 time points) (Figure 3). For each time point of a signal sample, observations during the past $120 \mathrm{~s}$ (4000 points) were used to construct a learning set and a predictor is formed to forecast the next $0.3 \mathrm{~s}$ (10 points) given the previous $3 \mathrm{~s}$ (100 points). In detail, let $N=4000$, $n=100, m=10$, and $\left\{x_{0}, \ldots, x_{M-1}\right\}$ denote a signal sample, where $M=10000$. For each $t=N+n, \ldots, M-m$, the signal tuple $\left(x_{t-n-N}, \ldots, x_{t-n-1}\right) \in \mathbb{R}^{N}$ was used to construct a learning set $\mathscr{L}_{t} \subset \mathbb{R}^{n} \times \mathbb{R}^{m}$ as in Section 2.3. Then, $\Psi_{\mathscr{L}_{t}}\left(\mathbf{x}_{t}\right) \in \mathbb{R}^{m}$ was calculated (Section 2.1), where $\mathbf{x}_{t}=\left(x_{t-n}, \ldots, x_{t-1}\right)$. To evaluate the prediction accuracy, the $m$ th coordinate of $\Psi_{\mathscr{L}_{t}}\left(\mathbf{x}_{t}\right)$, denoted as $\hat{x}_{t+m-1}$, was compared with the corresponding actual observation $x_{t+m-1}$. In accordance with the previous studies of predicting respiratory motion [7], the root mean square error (RMSE) (mm)

$$
\sqrt{\frac{\sum_{i=N+n+m-1}^{M-1}\left(\widehat{x}_{i}-x_{i}\right)^{2}}{M-N-n-m+1}}
$$

was calculated as an indicator of prediction error (Figure 4). The signal samples with RMSE less than $1.5 \mathrm{~mm}$ appeared to be well predictable by our approach (Figure 5), while some of the others appeared not to (Figure 6). Hence, the former samples numbered $0,1,2,7$, and 8 were selected for the next experiment.

3.3. Prediction-Based RGRT. Our prediction-based gating system, pRGRT, was tested using the selected five signal samples. In the following experiment, gate on and off delays were set to be $0.336 \mathrm{~s}$ and $0.088 \mathrm{~s}$, respectively, in accordance with the Abches system (Table 1). For each time point 

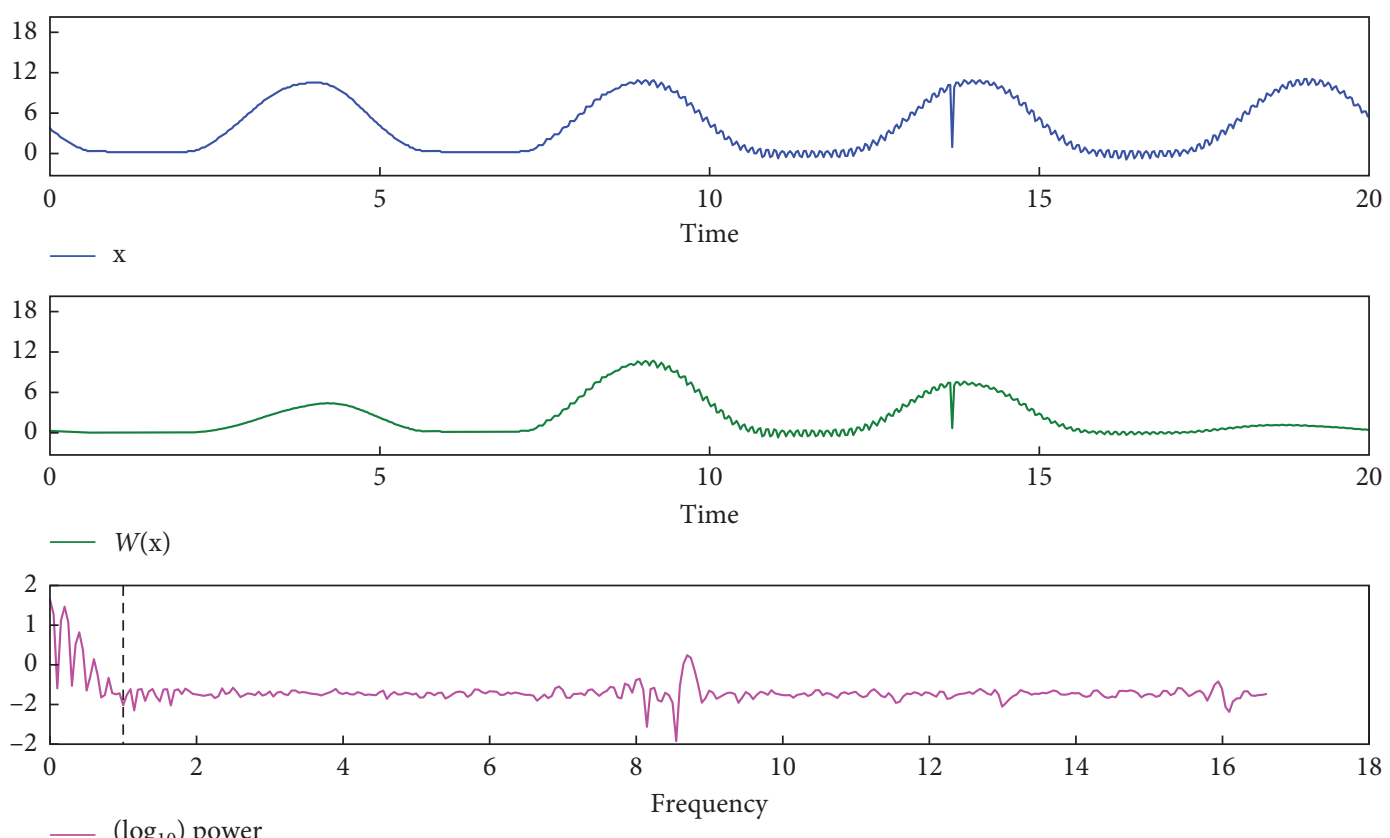

$\begin{array}{ll}- & \left(\log _{10}\right) \text { power } \\ ---\alpha & \end{array}$

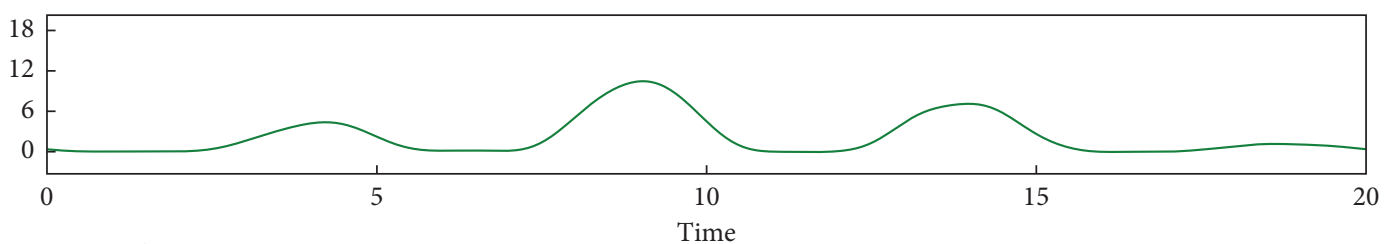

$-F_{N}^{-1} F_{\alpha} F_{N} W(\mathrm{x})$

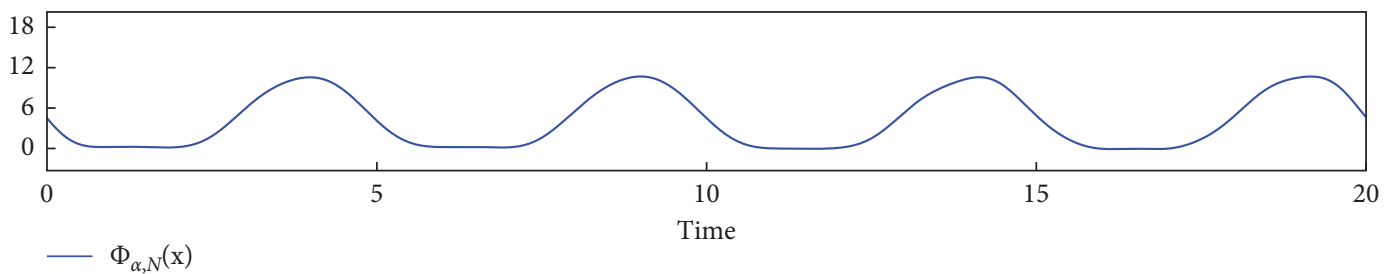

Figure 2: Smoothing of a respiratory signal. The phantom's signal was measured for $20 \mathrm{~s}$, and an artificial noise was added (13.65-13.7 s), forming a signal tuple $\mathbf{x}=\left(x_{0}, \ldots, x_{N-1}\right)$. Then $\Phi_{\alpha, N}(\mathbf{x})$ was calculated (Equations (8a)-(8d)), setting $\alpha=N \Delta \tau$ to filter out high frequency $(>1 \mathrm{~Hz})$ components. Note that power indicates $\left(\left|s_{0}\right|, \ldots,\left|s_{\mid(N-1) / 2}\right|\right)$, where $\left(s_{0}, \ldots, s_{N-1}\right)=\mathscr{F}_{N}\left(W\left(\left(x_{0}, \ldots, x_{N-1}\right)\right)\right)$, $|s|$ denotes the absolute value of $s \in \mathbb{C}^{1}$, and $\lfloor a\rfloor$ is the largest integer smaller than or equal to $a \in \mathbb{R}^{1}$. The units of signal value, time, and frequency are mm, $\mathrm{s}$, and $\mathrm{Hz}$, respectively.

$t \geq N+n$ of a sample $\left\{x_{0}, \ldots, x_{M-1}\right\}$, the signal tuple $\left(x_{t-n-N}, \ldots, x_{t-n-1}\right) \in \mathbb{R}^{N}$ was used to construct learning sets $\mathscr{L}_{1, t} \subset \mathbb{R}^{n} \times \mathbb{R}^{2 m_{1}+1}$ and $\mathscr{L}_{0, t} \subset \mathbb{R}^{n} \times \mathbb{R}^{2 m_{0}+1}$ as in Section 2.3, where $M=10000$ (300 s), $N=4000$ (120 s), $n=100$ (3s), $m_{1}=12(0.336 \mathrm{~s})$, and $m_{0}=3(0.088 \mathrm{~s})$. We put $\left\{g_{j}\right\}$ and $\left\{\widehat{g}_{j}\right\}$ as in Algorithm 1 and Algorithm 2, respectively, where $\beta$ was fixed to the median of $\left\{x_{0}, \ldots, x_{N-1}\right\}$.

For $j \in S=\left\{N+n+m_{1}-1, \ldots, M-1\right\}$, we assumed that gate on command is executed at $j$,

(i) if and only if $g_{j}=1$ (in conventional RGRT).

(ii) if and only if $\hat{g}_{j}=1$ (in pRGRT).

In each of the RGRT simulations, let $S_{1}$ be the set of $j \in S$ at which gate on command is executed, and put $S_{0}=S \backslash S_{1}$. To quantify possibly inappropriate irradiation during RGRT, the value

$$
\frac{\sum_{j \in S}\left(\chi_{S_{1}}(j) x_{j}^{+}+\chi_{S_{0}}(j) x_{j}^{-}\right)}{M-N-n-m_{1}+1},
$$

was calculated and denoted as nErr (normalized error), whose unit is $\mathrm{mm}$. Here, $\chi_{S}$ represents the characteristic function of a set $S$ defined as

$$
\chi_{S}(j)= \begin{cases}1, & \text { if } j \in S, \\ 0, & \text { if } j \notin S,\end{cases}
$$

$x_{j}^{+}=\max \left\{x_{j}-\beta, 0\right\}$, and $x_{j}^{-}=-\min \left\{x_{j}-\beta, 0\right\}$. Schematic illustrations of nErr and pRGRT are shown in Figure 7. As a result, nErr values for four out of the five samples 

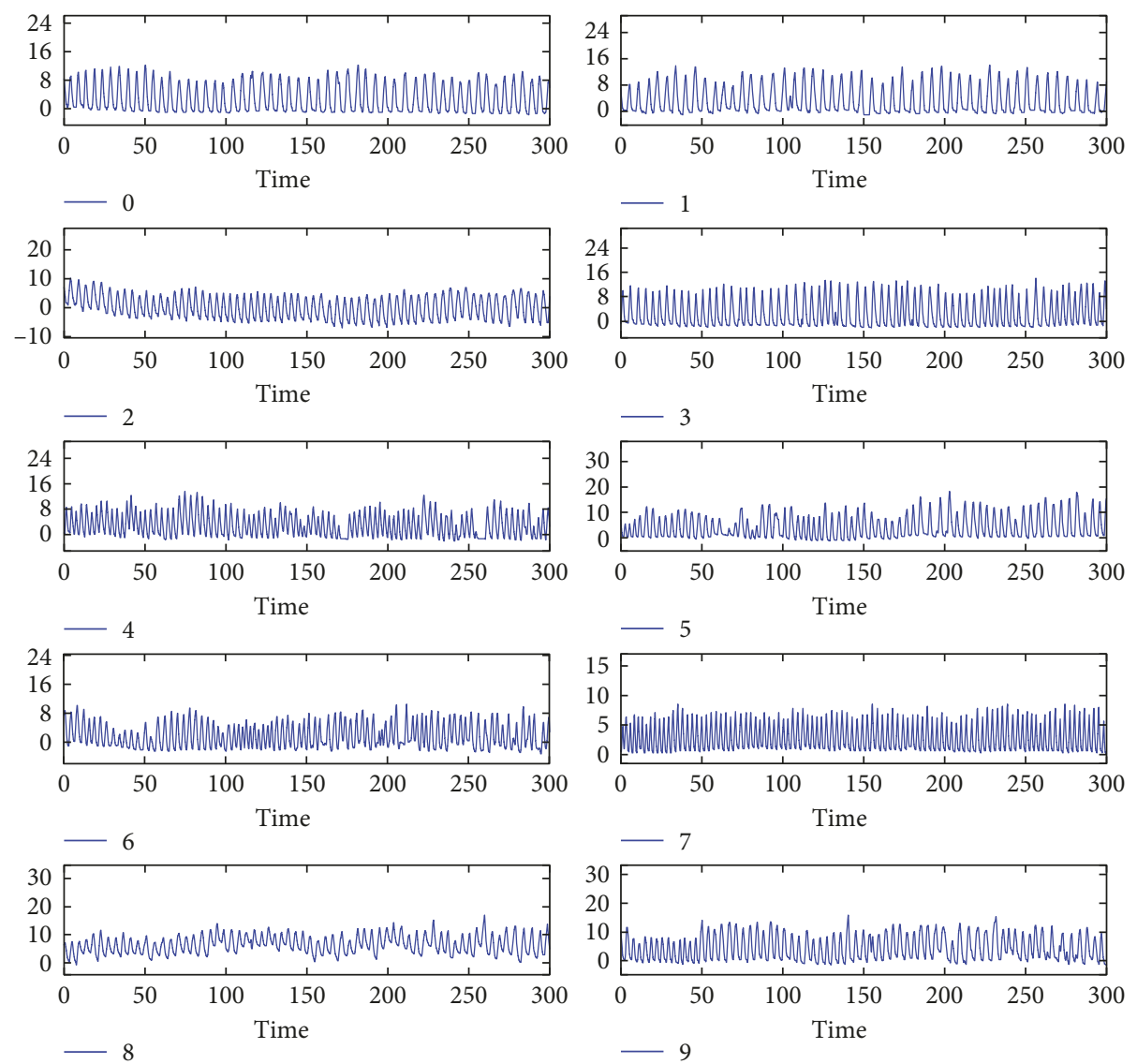

FIGURE 3: Respiratory signal samples of ten volunteers measured for $300 \mathrm{~s}$. The units of signal value and time are mm and s, respectively.

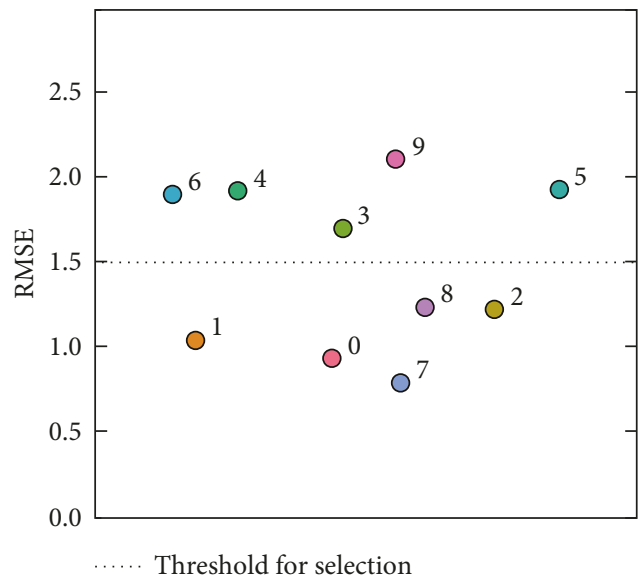

Figure 4: Prediction errors for the ten samples. For each sample, RMSE (mm) value was calculated (Section 3.2) and is plotted here.

decreased in pRGRT (Figure 8). Regarding the four samples, gating window shifts observed in conventional RGRT appeared to be improved in pRGRT (Figure 9). As for the other sample (numbered 8), considerable baseline drift was observed (Figure 10), which is an undesirable feature for gating systems with fixed threshold [12].
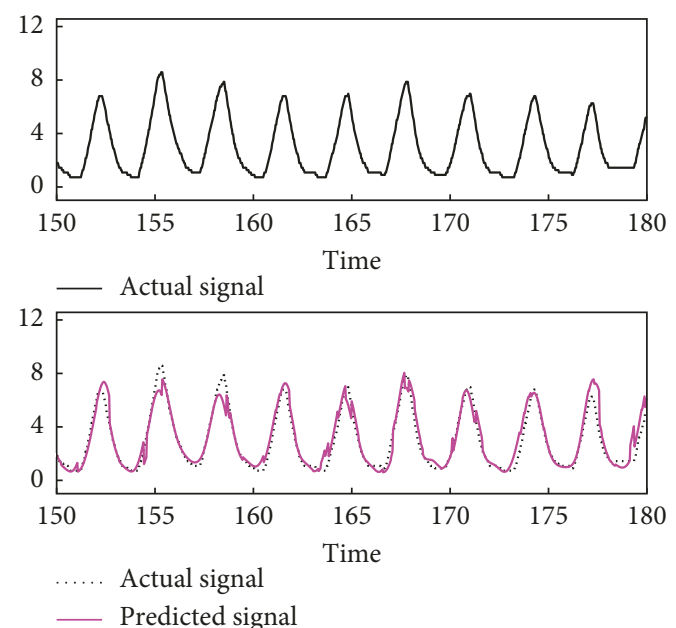

Figure 5: Representative prediction result. Predicted signal corresponds to $\widehat{x}_{5000}, \ldots, \widehat{x}_{5999}$ of the serial prediction trials (Section 3.2) using the sample numbered 7. The units of signal value and time are $\mathrm{mm}$ and $\mathrm{s}$, respectively.

The above are cases where $m_{1} \geq m_{0}$. To see whether pRGRT works when $m_{1}<m_{0}$, similar simulations were performed with gate on and off delays being $0.356 \mathrm{~s}$ $\left(m_{1}=12\right)$ and $0.529 \mathrm{~s} \quad\left(m_{0}=18\right)$, respectively, in 

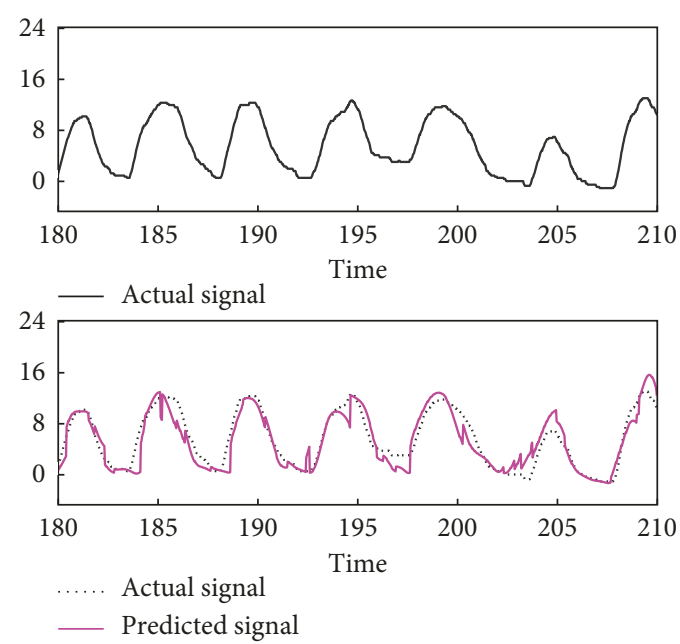

FIGURE 6: Representative prediction result. Predicted signal corresponds to $\widehat{x}_{6000}, \ldots, \widehat{x}_{6999}$ of the serial prediction trials (Section 3.2 ) using the sample numbered 9 . The units of signal value and time are $\mathrm{mm}$ and $\mathrm{s}$, respectively.

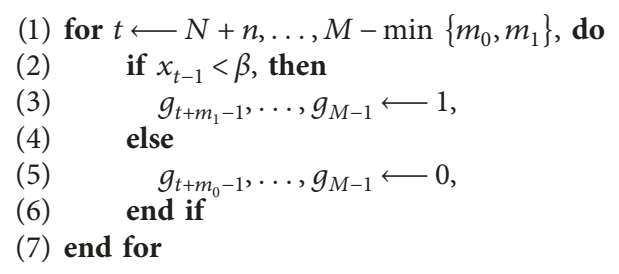

Algorithm 1: Simulation of conventional RGRT.

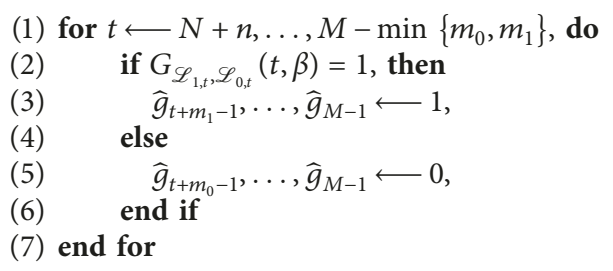

Algorithm 2: Simulation of pRGRT.

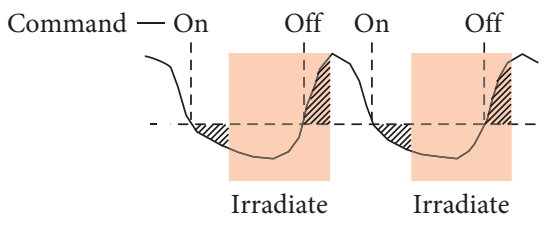

(a)

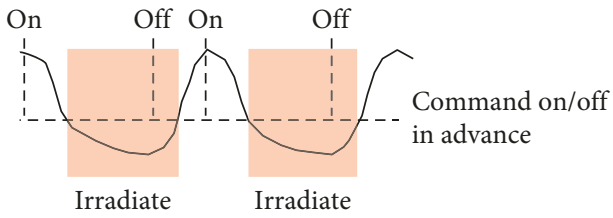

(b)

FIGURE 7: Conventional and prediction-based RGRT systems, denoted here as RGRT and pRGRT, respectively. (a) In RGRT, gate on and off delays cause shifts of gating windows. Stated informally, nErr corresponds to the mean absolute height of the shaded area (mm). (b) In pRGRT, gate on and off commands are expected to be sent in advance to compensate for the latencies. 


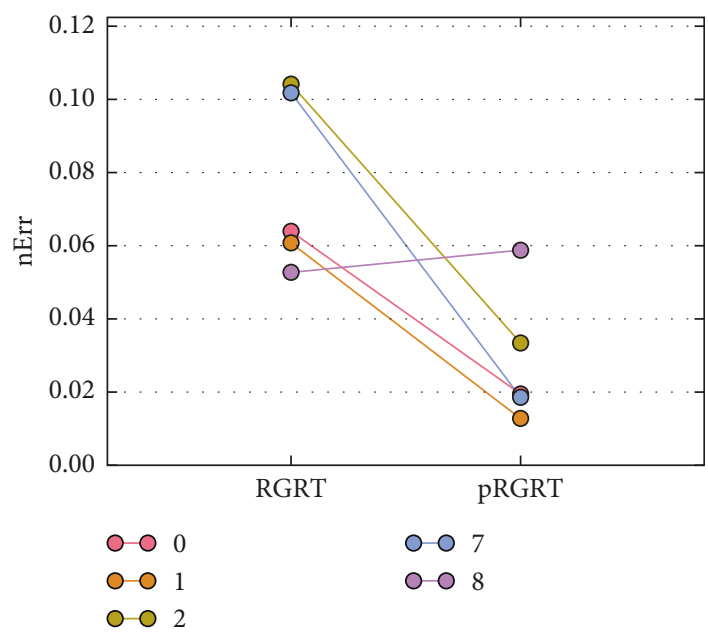

FIGURE 8: nErr values for the selected five samples in the conventional and prediction-based RGRT simulations (denoted here as RGRT and pRGRT, respectively) mimicking the Abches system. The unit of nErr is $\mathrm{mm}$.
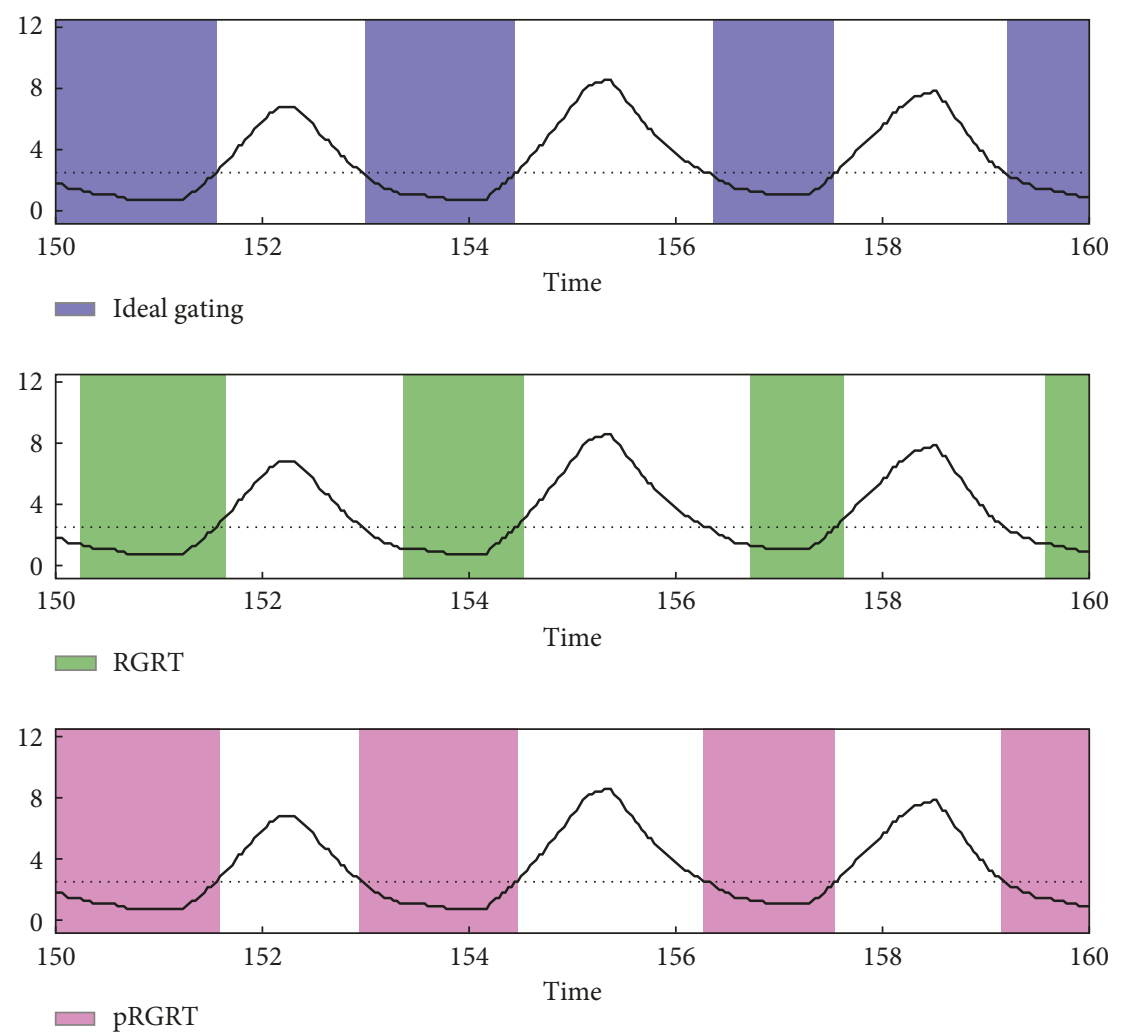

FIGURE 9: Gating windows in the conventional and prediction-based RGRT simulations (denoted here as RGRT and pRGRT, respectively) which mimic the Abches system, using the sample numbered 7. The colored rectangles of RGRT and pRGRT correspond to $\left\{j: g_{j}=1\right\}$ and $\left\{j: \widehat{g}_{j}=1\right\}$, respectively, where $5000 \leq j \leq 5333$. The units of signal value and time are $\mathrm{mm}$ and $\mathrm{s}$, respectively.

accordance with the the AlignRT system (Table 1). The outcome was that nErr values for all the samples decreased in pRGRT (Figure 11) and gating window shifts in conventional RGRT were ameliorated in pRGRT (Figure 12).

\section{Conclusions}

In this paper, we proposed a framework to compensate for gate on/off latency during RGRT. It consisted of two steps: (1) multistep-ahead prediction and (2) prediction-based gating. 

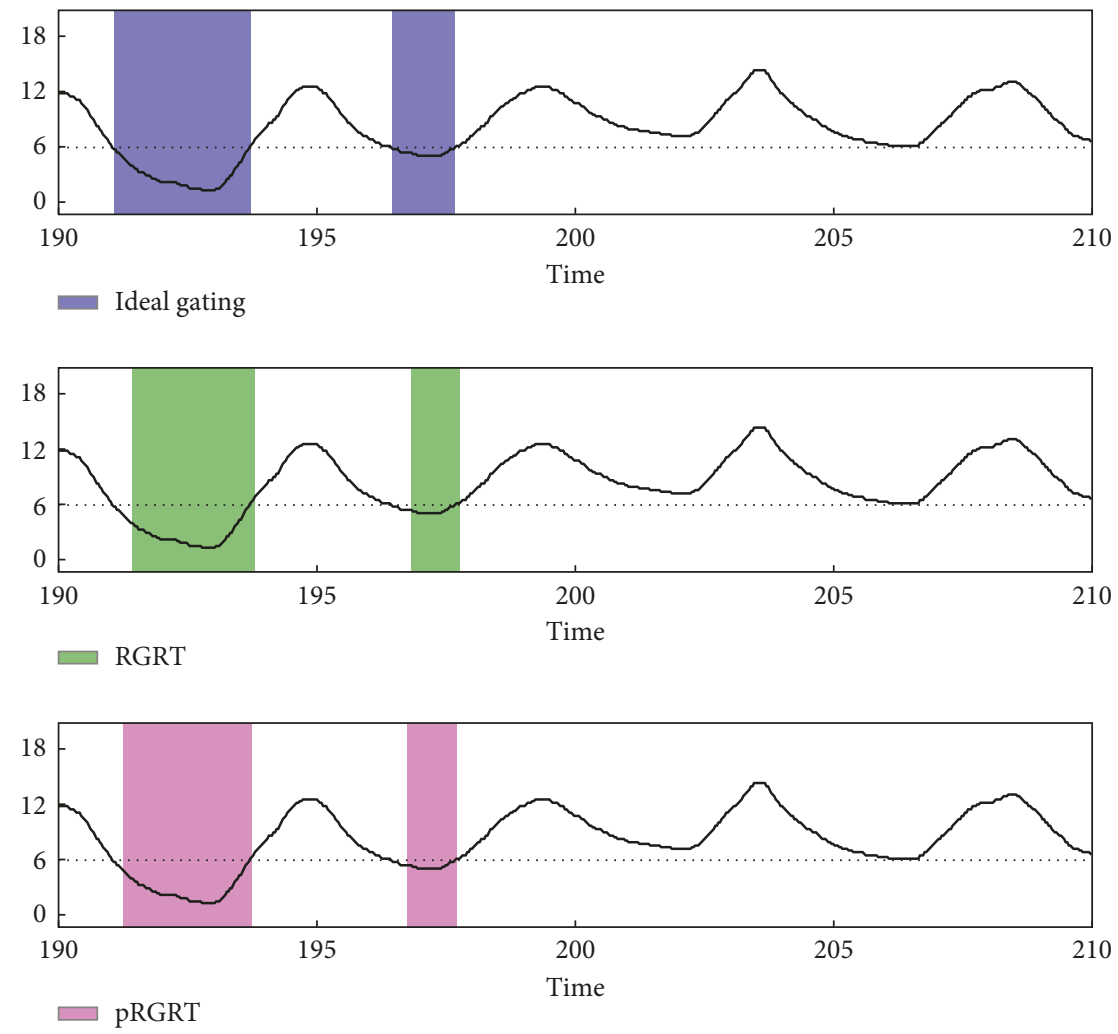

FIGURE 10: Gating windows in the conventional and prediction-based RGRT simulations (denoted here as RGRT and pRGRT, respectively) which mimic the Abches system, using the sample numbered 8. The colored rectangles of RGRT and pRGRT correspond to $\left\{j: g_{j}=1\right\}$ and $\left\{j: \widehat{g}_{j}=1\right\}$, respectively, where $6334 \leq j \leq 6999$. The units of signal value and time are $\mathrm{mm}$ and $\mathrm{s}$, respectively.

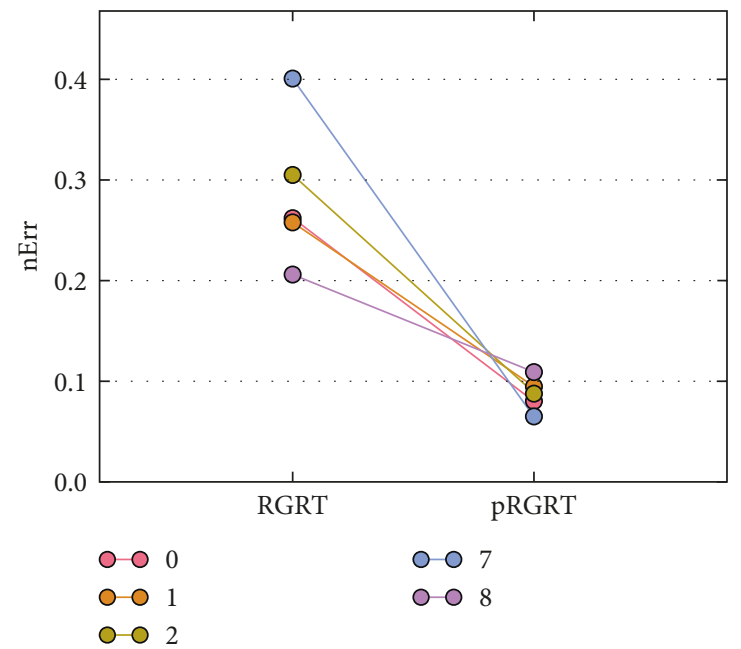

FIGURE 11: nErr values for the selected five samples in the conventional and prediction-based RGRT simulations (denoted here as RGRT and pRGRT, respectively) mimicking the AlignRT system. The unit of nErr is mm.

For each step, we devised a specific algorithm to accomplish the task. Numerical experiments were performed using respiratory signals of a phantom and ten volunteers, and our predictionbased RGRT system, pRGRT, displayed superior performance in not a few of the signal samples. In some, however, signal prediction and prediction-based gating did not work well, probably because of signal irregularity and/or baseline drift.
The developed method has potential applicability in RGRT, but there are several issues to be addressed, e.g.,

(1) Are there better algorithms for multistep-ahead prediction?

(2) Are there better algorithms for prediction-based gating?

(3) Is it possible to deal with baseline drift? 

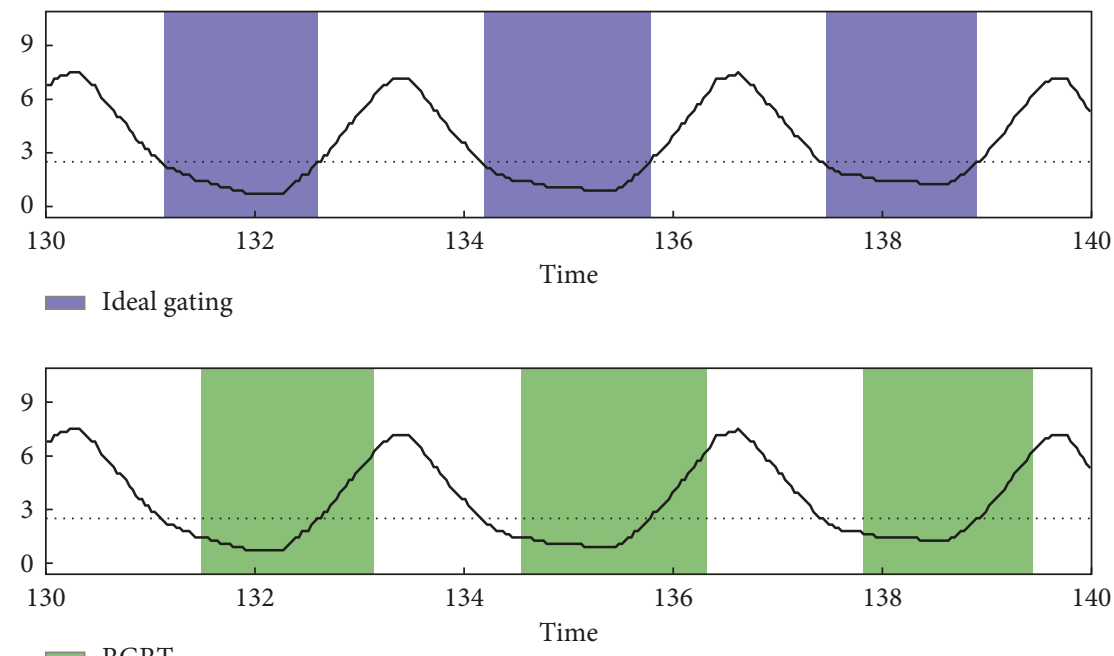

RGRT

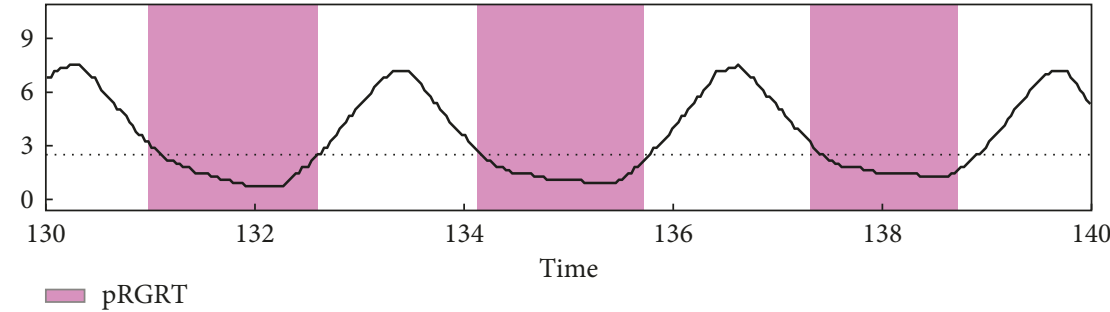

FIGURE 12: Gating windows in the conventional and prediction-based RGRT simulations (denoted here as RGRT and pRGRT, respectively) which mimic the AlignRT system, using the sample numbered 7. The colored rectangles of RGRT and pRGRT correspond to $\left\{j: g_{j}=1\right\}$ and $\left\{j: \widehat{g}_{j}=1\right\}$, respectively, where $4334 \leq j \leq 4666$. The units of signal value and time are mm and s, respectively.

(4) Is it possible to provide theoretical foundations to the methods?

(5) Is the method valid in a real clinical setting?

Further studies on these matters would be needed for the system to be of practical use.

\section{Data Availability}

The respiratory signal data used in the current study are available in the Figshare repository (https: //doi.org/10.6084/m9.figshare.6290924).

\section{Conflicts of Interest}

The authors declare no conflicts of interest.

\section{Acknowledgments}

This work was funded by APEX Medical, Inc. (Tokyo, Japan). We would like to thank Kazunori Nakamoto (University of Yamanashi) for carefully proofreading a draft of this paper. We are grateful to Editage (http: //www.editage.jp) for English language editing.

\section{References}

[1] H. D. Kubo and B. C. Hill, "Respiration gated radiotherapy treatment: a technical study," Physics in Medicine and Biology, vol. 41, no. 1, pp. 83-91, 1996.
[2] S. S. Vedam, P. J. Keall, V. R. Kini, and R. Mohan, "Determining parameters for respirationgated radiotherapy," Medical Physics, vol. 28, no. 10, pp. 2139-2146, 2001.

[3] P. J. Keall, G. S. Mageras, J. M. Balter et al., "The management of respiratory motion in radiation oncology report of AAPM Task Group 76a)," Medical Physics, vol. 33, no. 10, pp. 3874-3900, 2006.

[4] M. Saito, N. Sano, K. Ueda et al., "Technical note: evaluation of the latency and the beam characteristics of a respiratory gating system using an elekta linear accelerator and a respiratory indicator device, Abches," Medical Physics, vol. 45, no. 1, pp. 74-80, 2018.

[5] R. D. Wiersma, B. P. McCabe, A. H. Belcher, P. J. Jensen, B. Smith, and B. Aydogan, "Technical note: high temporal resolution characterization of gating response time," Medical Physics, vol. 43, no. 6, pp. 2802-2806, 2016.

[6] P. Freislederer, M. Reiner, W. Hoischen et al., "Characteristics of gated treatment using an optical surface imaging and gating system on an elekta linac," Radiation Oncology, vol. 10, no. 1, p. 68,2015 .

[7] S. J. Lee and Y. Motai, Review: Prediction of Respiratory Motion, Springer Berlin Heidelberg, Berlin, Heidelberg, 2014.

[8] S. B. Taieb, A. Sorjamaa, and G. Bontempi, "Multiple-output modeling for multi-step-ahead time series forecasting," Neurocomputing, vol. 73, pp. 1950-1957, 2010.

[9] P. J. Nicholson, "Algebraic theory of finite fourier transforms," Journal of Computer and System Sciences, vol. 5, no. 5, pp. 524-547, 1971

[10] P. Stoica and R. Moses, Spectral Analysis of Signals, Pearson Prentice Hall, Upper Saddle River, NJ, USA, 2005. 
[11] H. Onishi, H. Kawakami, K. Marino et al., "A simple respiratory indicator for irradiation during voluntary breath holding: a one-touch device without electronic materials," Radiology, vol. 255, no. 3, pp. 917-923, 2010.

[12] E. W. Pepin, H. Wu, and H. Shirato, "Dynamic gating window for compensation of baseline shift in respiratorygated radiation therapy," Medical Physics, vol. 38, no. 4, pp. 1912-1918, 2011. 


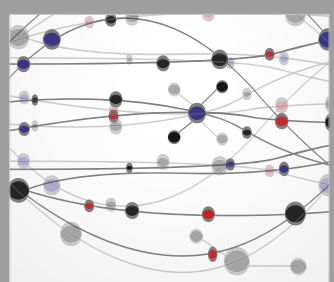

The Scientific World Journal
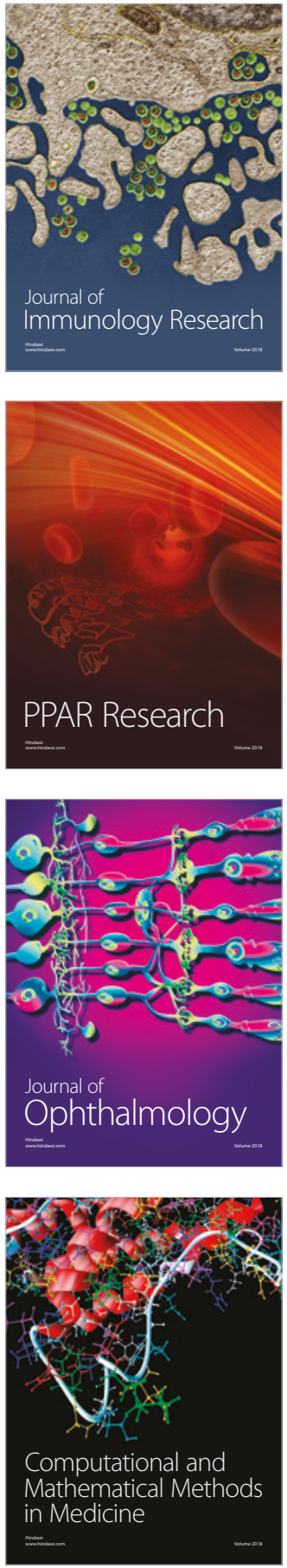

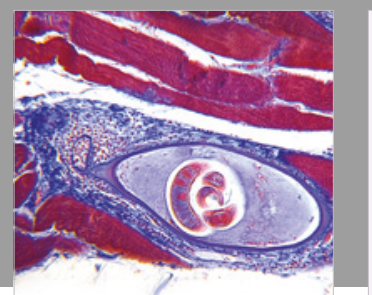

Gastroenterology Research and Practice

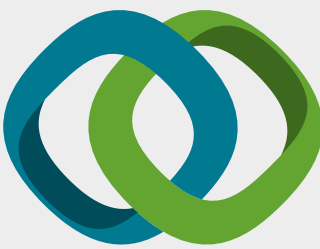

\section{Hindawi}

Submit your manuscripts at

www.hindawi.com
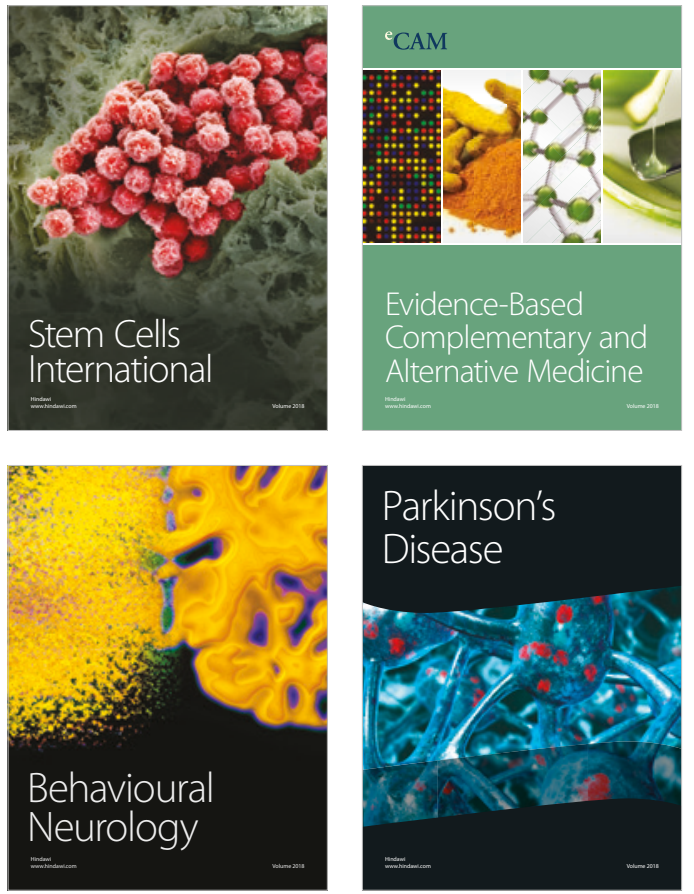

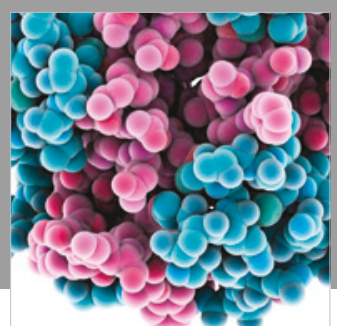

ournal of

Diabetes Research

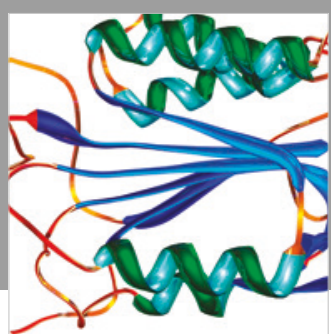

Disease Markers
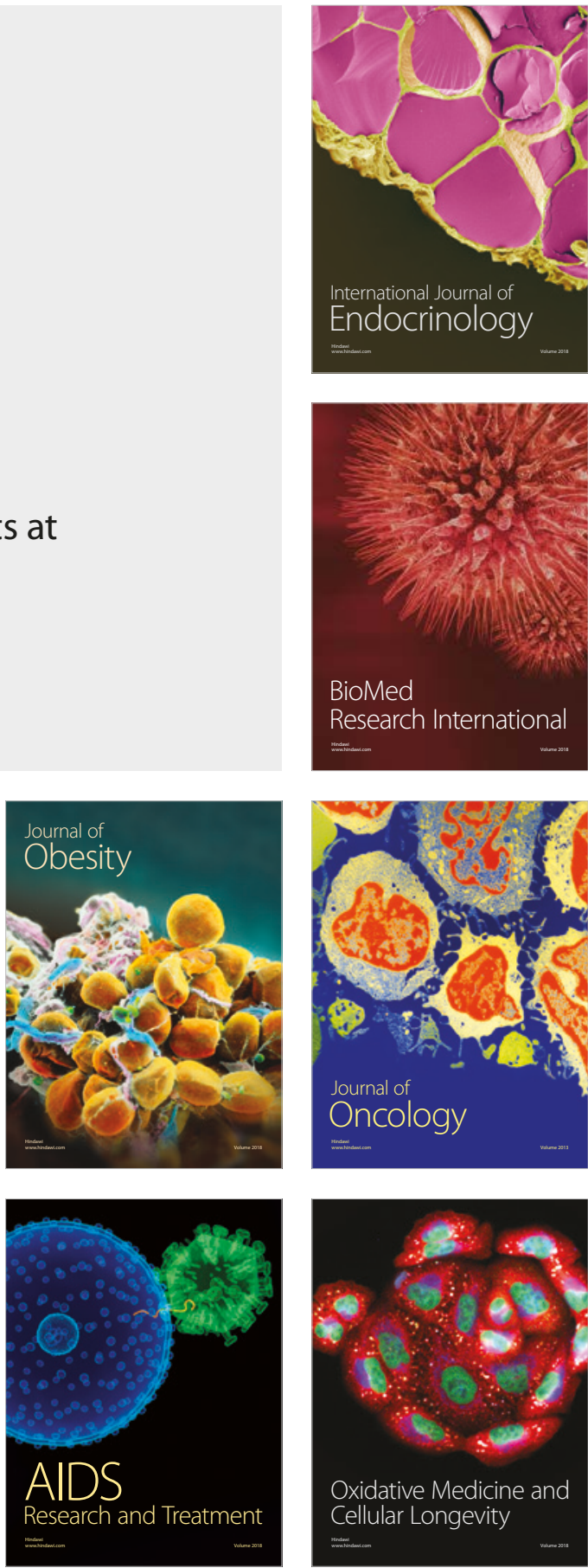